\title{
Numerical Solution of Fractional Benney Equation
}

\author{
Mehmet Ali Akinlar, Aydin Secer and Mustafa Bayram* \\ Yildiz Technical University, Faculty of Chemical and Metallurgical Engineering, Department of Mathematical Engineering, 34210- \\ Davutpasa-Istanbul, Turkey
}

Received: 19 Jul. 2013, Revised: 21 Oct. 2013, Accepted: 22 Oct. 2013

Published online: 1 Jul. 2014

\begin{abstract}
In this paper we propose a new solution technique for numerical solution of fractional Benney equation, a fourth degree nonlinear fractional partial differential equation with broad range of applications. The method could be described as a hybrid technique which uses advantages of both wavelets and operational matrices. Having applied the present method, fractional Benney equation is converted into a matrix equation, which is easy to solve. To the best of our knowledge, the fractional Benney equation has not been solved with any numerical or analytical method in the literature. Solving this equation numerically and investigating the applicability of the wavelets on this problem is the main goal of this paper. Haar wavelets and Caputo type fractional derivatives are employed in the calculations. Computational results point the strength of Haar wavelets and feasibility of the present solution algorithm.
\end{abstract}

Keywords: Fractional Benney equation, Haar wavelets, numerical solution, Caputo fractional derivative.

\section{Introduction}

Fractional calculus is one of the most popular calculus types having a vast range of applications in many different scientific and engineering disciplines. Order of the derivatives in the fractional calculus might be any reel number which separates the fractional calculus from the ordinary calculus where the derivatives are allowed to be only natural numbers. Fractional calculus is a highly efficient and useful tool in the modeling of many sorts of scientific phenomena including image processing, earthquake engineering, biomedical engineering, computational fluid mechanics and Physics. Fundamental concepts of fractional calculus and applications of it to different research areas can be seen in the references, [1], [2], [8], [10], [13] and [14] (amongst many others).

In this paper we employ a hybrid technique which uses advantages of both wavelets and fractional calculus for the numerical solution of the fractional Benney equation. The present numerical method could be described as unification of wavelets with fractional calculus which obtains the solution in a highly simple and efficient manner. To the best of our knowledge, the fractional Benney partial differential equation has not been solved with the present method, solution of this equation with a new method is the main goal of this paper. Another important point to mention inhere is that applicability of the wavelet techniques to the fractional differential equations have not been studied in detail in the literature. Therefore, investigating the applications of wavelet based methods to the numerical solution of such a high order and nonlinear partial differential equation shall be a significant contribution to the subject.

Fractional calculus is a quite useful and significantly important subject which usually involves challenging problems, efficient solution algorithms, fast solvers and high storage requirements. Nevertheless, a general solution algorithm or technique which could be employed at almost every sorts of problems has not yet been established. Each solution method has been developed for particular types of problems. As a consequence, a single standard method for problems regarding fractional calculus has not emerged. Therefore, finding reliable and efficient solution techniques along with fast implementation methods are useful and active research areas. Some well known methods for the analytical and numerical solutions of fractional differential and integral equations might be listed as power series method [11], differential transform method [3] and [9], homotopy analysis method [4], variational iteration method [5] and homotopy perturbation method [6]. Typical numerical methods including collocation, finite differences and elements are among the most popular numerical techniques and detailed information about most of which

\footnotetext{
* Corresponding author e-mail: msbayram@yildiz.edu.tr
} 
can be obtained from the aforementioned references and their own references.

Deterministic Benney partial differential equation is defined as

$$
u_{t}+\left(u^{n}\right)_{x}+u_{x x}+k u_{x x x}+u_{x x x x}=0,
$$

where $k$ is a constant real number and $n$ is a positive integer number. Considering $n=3$, we can write 1 as

$$
u_{t}+3 u^{2} u_{x}+u_{x x}+k u_{x x x}+u_{x x x x}=0 .
$$

Benney equation has a broad range of applications at solitons theory, computational fluid dynamics and Physics. Some applications regarding the Benney equation can be seen, for example, at [?].

In this paper, we are particularly concerning with the numerical solution of fractional Benney equation defined as a perturbation of the time derivative at (2). Fractional Benney equation that we consider in this paper is

$$
D_{*_{t}}^{\alpha} u+3 u^{2} u_{x}+u_{x x}+k u_{x x x}+u_{x x x x}=0 .
$$

This paper consists of four major parts. After the present introduction section, in the second and third sections we briefly overview some basic definitions and theorems regarding wavelets and fractional calculus, respectively. In section 4 we solve the equation (3) numerically in an efficient manner. We complete the paper with a discussion part where we summarize the present paper and mention some future extensions.

\section{Wavelets}

Wavelets developed in the beginning of the last century is a highly useful and efficient method in signal processing and computational mathematics. Wavelet theory involves representing functions via simple building blocks at different scales and positions. The fundamental idea behind wavelets is to analyze according to scale. Some of the most popular wavelet types are Haar, Daubechies, Shannon, Coiflet, spline, Battle-Lemaries, some orthogonal wavelets such as Legendre, Hermite and Chebyshev.

In the last decade wavelets have been employed in the solutions of differential equations. The structure of wavelets makes them quite appropriate for solving the partial differential equations. Typical wavelet based computational techniques employ some basis functions which are differentiable and do not vanish on the compact support. Some other related methods might be listed as finite difference methods (FDM), finite volume methods (FVM), finite elements methods (FEM), spectral methods. FDM and FVM are an approximation to the differential equation while other methods are an approximation to its solution. Even though the spectral bases are infinitely differentiable, they have global support which could be considered as a negative point. Wavelets have the capability of representing the solutions at different scales, which make them particularly useful for developing efficient solutions to the approximate solutions of partial differential equations and fractional differential equations.

A function $\psi \in L^{2}(\mathbb{R})$ is said to be a wavelet if

$$
\int_{-\infty}^{\infty} \psi(t) d t=0
$$

Notice that if $\psi \in L^{2}(\mathbb{R}) \bigcap L^{1}(\mathbb{R})$ that satisfies

$$
c_{\psi}=2 \pi \int_{-\infty}^{\infty} \frac{|\widehat{\psi}(w)|^{2}}{|w|} d w \leq \infty
$$

where $\widehat{\psi}(w)$ is the Fourier transform of $\psi$, then (4) holds.

Lemma: Let $\psi \in L^{2}(\mathbb{R})$ have a compact support. The the following two statements are equivalent.

(i.) $\psi$ is a wavelet.

(ii.)The equality (5) is satisfied.

In the literature there are so many different types of wavelets. In this paper we are interested in an orthogonal wavelet having a compact support, namely, Haar wavelet that could be defined as follows:

Define

$$
\psi(x)= \begin{cases}1, & 0 \leq x<1 / 2 \\ -1, & 1 / 2 \leq x<1 \\ 0, & \text { otherwise }\end{cases}
$$

Is is clear that $\psi(x)$ satisfies (4) and $\psi(x)$ has a compact support on $[0,1]$. It is not hard to prove that $\psi(x)$ also satisfies (5). $\psi(x)$ defined in this way is known as Haar wavelet developed by A. Haar in 1909.

Discrete versions of continuous wavelets might be defined as follows. A function $\psi \in L_{2}(\mathbb{R})$ is a discrete wavelet if the family of functions $\psi_{j, k}(t)$ defined by

$$
\psi_{j, k}(t)=2^{j / 2} \psi\left(2^{j} t-k\right)
$$

where $j$ and $k$ are arbitrary integers, is an orthonormal basis in the Hilbert space $L_{2}(\mathbb{R})$.

Wavelet coefficients of a function $f \in L_{2}(\mathbb{R})$, denoted by

$$
d_{j, k}=\int_{\mathbb{R}} f(t) \psi_{j, k}(t) d t
$$

The series

$$
\sum_{j, k \in \mathbb{Z}} \psi_{j, k}(t) \int_{\mathbb{R}} f(t) \psi_{j, k}(t) d t
$$

is called the wavelet series of $f$. The expression

$$
f=\sum_{j, k \in \mathbb{Z}} \psi_{j, k}(t) \int_{\mathbb{R}} f(t) \psi_{j, k}(t) d t
$$


is called the wavelet representation of $f$.

In the application section we shall illustrate an approximation to a $u(x, t) \in L^{2}([0,1) \times[0,1))$ function with Haar wavelets, therefore we leave the information regarding the applications by Haar wavelets to functions to the main section. For detailed and further properties of wavelets and particularly Haar wavelets, interested reader can read, for instance, [7] in conjunction with the present paper.

\section{Fractional calculus}

In this section, we briefly overview the some fundamental concepts of fractional calculus. As we mentioned in the introductory part, orders of derivatives and integrals in fractional calculus might be at any real number. The most popular definitions of a fractional derivative of a function are Riemann-Liouville, Grunwald-Letnikow, Caputo and Generalized functions. In this paper Caputo's definition of fractional differentiation will be employed.

Definition. A real function $f(x), x>0$, is said to be in the space $C_{\rho}, \rho \in R$ if there exists a real number ( $p>$ $\rho$ ), such that $f(x)=x^{p} f_{1}(x)$ for a continuous function $f_{1}(x) \in C[0, \infty)$.

Definition. The Riemann-Liouville fractional integral operator of order $\alpha \geq 0$ of a function $f \in C_{\rho}, \rho \geq-1$, is defined as

$$
\begin{gathered}
J_{0}^{v} f(x)=\frac{1}{\Gamma(v)} \int_{0}^{x}(x-t)^{v-1} f(t) d t, \quad v>0, \\
J^{0} f(x)=f(x) .
\end{gathered}
$$

It has the following properties:

For $f \in C_{\rho}, \rho \geq-1, \alpha, \beta \geq 0$ and $\gamma>1$ :

$$
\begin{aligned}
& \text { i.) } J^{\alpha} J^{\beta} f(x)=J^{\alpha+\beta} f(x), \\
& \text { ii.) } J^{\alpha} J^{\beta} f(x)=J^{\beta} J^{\alpha} f(x), \\
& \text { iii.) } J^{\alpha} x^{\gamma}=\frac{\Gamma(\gamma+1)}{\Gamma(\alpha+\gamma+1)} x^{\alpha+\gamma} .
\end{aligned}
$$

Next we present the Caputo sense derivative.

Definition. The fractional derivative of $f(x)$ in the Caputo sense is defined as

$$
D_{*}^{v} f(x)=\frac{1}{\Gamma(m-v)} \int_{0}^{x}(x-t)^{m-v-1} f^{(m)}(t) d t,
$$

for $m-1<v<m, m \in N, x>0, f \in C_{-1}^{m}$.

Definition. For $m$ to be the smallest integer that exceeds $\alpha$, the Caputo time-fractional derivative operator of order $\alpha>0$ is defined as $D_{* t}^{\alpha} u(x, t)=$

$$
\left\{\begin{array}{lc}
\frac{1}{\Gamma(m-\alpha)} \int_{0}^{t}(t-\xi)^{m-\alpha-1} \frac{\partial^{m} u(x, \xi)}{\partial \xi^{m}} d \xi, & -1<\alpha<m \\
\frac{\partial^{m} u(x, t)}{\partial t^{m}}, & \alpha=m \in N
\end{array}\right.
$$

and the space-fractional derivative operator of order $\beta>0$ is defined as $D_{* x}^{\alpha} u(x, t)=$

$$
\left\{\begin{array}{l}
\frac{1}{\Gamma(m-\beta)} \int_{0}^{x}(x-\theta)^{m-\beta-1} \frac{\partial^{m} u(\theta, t)}{\partial \theta^{m}} d \theta, m-1<\beta<m, \\
\frac{\partial^{m} u(x, t)}{\partial x^{m}}, \\
\beta=m \in N .
\end{array}\right.
$$

Lemma. If $m-1<\alpha<m, m \in N$ and $f \in C_{\rho}^{m}, \rho \geq$ -1 , then

$$
\begin{aligned}
& D_{*}^{\alpha} J^{\alpha} f(x)=f(x), \\
& J^{\alpha} D_{*}^{v} f(x)=f(x)-\sum_{k=0}^{m-1} f^{k}\left(0^{+}\right) \frac{x^{k}}{k !}, \mathrm{x}>0 .
\end{aligned}
$$

The Caputo fractional derivative is considered here because it allows traditional initial and boundary conditions to be included in the formulation of the problem. In the next section we solve (3).

\section{Main results: Numerical solution of fractional Benney equation}

Consider an orthonormal matrix $\Psi(\tau)$, approximate solution of $n$-times integral of $\Psi(\tau)$ is defined by

$$
\int_{0}^{t} \cdots \int_{0}^{t} \Psi(\tau)(d \tau)^{n} \cong \Upsilon_{\Phi}^{n} \Psi(t), n \in \mathbb{N}
$$

where $\Psi(t)=\left[\Phi_{0}(t), \cdots, \Phi_{m-1}(t)\right]^{T}$. Here, $\Phi_{i}(t), i=$ $0, \cdots m-1$ are orthogonal basis functions on the interval $[0,1), \Upsilon_{\Phi}$ is matrix representation for integral of $\Psi(t)$.

Let $u(x, t) \in L^{2}([0,1) \times[0,1)) . u(x, t)$ can be represented by Haar wavelet basis as

$$
u(x, t)=\sum_{i=0}^{m-1} \sum_{j=0}^{m-1} w_{i j} h_{i}(x) h_{j}(t),
$$

where the coefficients are defined as

$$
\begin{gathered}
w_{i j}:=\int_{0}^{1} u(x, t) h_{i}(x) d x \int_{0}^{1} u(x, t) h_{j}(t) d t \\
i, j=0,1, \cdots, m-1 .
\end{gathered}
$$

By taking step $\Delta=\frac{1}{m}$ of $x, t,(10)$ can be written in discrete form as

$$
U(x, t)=H^{T}(x) W H(t),
$$

where $W=\left[w_{i j}\right]_{m-1, m-1}$ is the coefficient matrix of $U(x, t)$ and $h_{i}(x)$ is the Haar wavelet basis consisting of the Haar wavelet matrix $H$. The corresponding operational matrix is defined in terms of the well-known and so useful block matrix as

$$
\Upsilon_{H}^{\alpha}=H \Upsilon_{B}^{\alpha} H^{T}
$$


where $\Upsilon_{B}^{\alpha}$ is the Block pulse function. The operational matrix for integration of the block pulse function is given by

$$
\Upsilon_{B_{m}}^{\alpha}:=\frac{1}{m}\left(\begin{array}{cccc}
1 / 2 & 1 & \cdots & 1 \\
0 & \cdots & \cdots & \cdots \\
\cdots & 0 & 1 / 2 & 1 \\
0 & \cdots & 0 & 1 / 2
\end{array}\right)_{m \times m} .
$$

Equipped with these machinery, we can write the approximations of each term in the (3) in the following way.

$$
\begin{aligned}
D_{*_{t}}^{\alpha} u=\frac{\partial^{\alpha} u}{\partial t^{\alpha}} & \approx \frac{\partial^{\alpha} U}{\partial t^{\alpha}}=H^{T}(x) W \frac{\partial^{\alpha}}{\partial t^{\alpha}} H(t) \\
& =H^{T}(x) W \Upsilon_{H}^{-\alpha} H(t), \\
u_{x}(x, t) & =\frac{\partial u(x, t)}{\partial x} \approx H^{T}(x)\left(\Upsilon_{H}^{-1}\right)^{T} W H(t), \\
u_{x x}(x, t) & =\frac{\partial^{2} u(x, t)}{\partial x^{2}} \approx H^{T}(x)\left(\Upsilon_{H}^{-2}\right)^{T} W H(t), \\
u_{x x x}(x, t) & =\frac{\partial^{3} u(x, t)}{\partial x^{3}} \approx H^{T}(x)\left(\Upsilon_{H}^{-3}\right)^{T} W H(t), \\
u_{x x x x}(x, t) & =\frac{\partial^{4} u(x, t)}{\partial x^{4}} \approx H^{T}(x)\left(\Upsilon_{H}^{-4}\right)^{T} W H(t),
\end{aligned}
$$

Let $\alpha=\frac{2}{3}$. Then, using the identities given at (11), we can express the fractional Benney equation (3) as follows:

$$
\begin{array}{r}
H^{T} W \Upsilon_{H}^{-2 / 3} H+3 H^{T} W H H^{T} W H H^{T}\left(\Upsilon_{H}^{-1}\right)^{T} W H+ \\
H^{T}\left(\Upsilon_{H}^{-2}\right)^{T} W H+k H^{T}\left(\Upsilon_{H}^{-3}\right)^{T} W H+ \\
H^{T}\left(\Upsilon_{H}^{-4}\right)^{T} W H=0 .
\end{array}
$$

Multiplying this equation with $H$ from left and $H^{T}$ from right, we obtain

$$
\begin{aligned}
& W \Upsilon_{H}^{-2 / 3}+ 3 W^{2}\left(\Upsilon_{H}^{-1}\right)^{T} W+\left(\Upsilon_{H}^{-2}\right)^{T} W+ \\
& k H\left(\Upsilon_{H}^{-3}\right)^{T} W+\left(\Upsilon_{H}^{-4}\right)^{T} W=0 .
\end{aligned}
$$

It is clear that (9) is a matrix equation which can be solved by any convenient matrix solver softwares including Mathematica, Maple and Matlab. In order to obtain $W$, one can obtain experimental results for different cases of the size of the operation matrix $m$ (such as $m=8,16,32,64,128)$, and different values of $t$ and $\alpha$.

\section{Conclusion and future plans}

In this paper we presented a new solution technique for numerical solution of fractional Benney equation. The method could be described as a hybrid technique which uses advantages of both wavelets and fractional calculus. In fact, the present method could be described as a wavelet Galerkin method that employs the techniques on multi resolution analysis and use the advantage of multiscale methods, which make them more powerful methods than traditional numerical techniques. Haar wavelets and Caputo type fractional derivatives are employed in the calculations. Computational results point the strength of Haar wavelets and the present solution algorithm. Employing some other orthogonal wavelets and investigating the applicability of the present method to some other nonlinear fractional (stochastic) differential and integral equations might be useful problem as a future extensions of the present method.

\section{References}

[1] I. Podlubny, Fractional differential equations, An introduction to fractional derivatives, fractional differential equations, some methods of their solutionand some of their applications. San Diego: Academic Press, (1999).

[2] R. Hilfer (Ed.), Applications of fractional calculus in physics, Academic pres, Orlando, (1999).

[3] A. Secer, M. A. Akinlar and A. Cevikel, Efficient Solutions of Systems Of Fractional PDEs By Differential Transform Method, Advances in Difference Equations DOI:10.1186/1687-1847-2012-188, 2012, (2012).

[4] S. J. Liao, The proposed homotopy analysis techniques for the solution of nonlinear problems. Ph.D. dissertation, Shanghai Jiao Tong University, Shanghai, (1992).

[5] Z. Odibat and S. Momani, Application of variational iteration method to nonlinear differential equations of fractional order, Int. J. Nonlin. Sci. Numer. Simul., 7, 1527 (2006).

[6] J. Biazar and H. Ghazvini, Exact solutions for non-linear Schrödinger equations by He's homotopy perturbation method, Physics Letters A, 366, 79-84 (2007).

[7] S. Mallat, A wavelet tour of signal processing, Academic Press, (2008).

[8] Y. Feng, X. Lin and S. Zhou and H. Li, Chaos in a fractional-order neutral differential system, Applied mathematics and information sciences (AMIS), 7, 233-238 (2013).

[9] M. Kurulay and M. Bayram, Approximate analytical solution for the fractional modified $K d V$ by differential transform method, Communications in nonlinear science and numerical simulation, 15, 1777-1782 (2010).

[10] M. Bayram, Automatic analysis of the control of metabolic networks, Computers in biology and medicine, 26, 401-408 (1996).

[11] E. Celik, E. Karaduman and M. Bayram, Numerical solutions of chemical differential-algebraic equations, Applied mathematics and computation, 139, 259-264 (2003).

[12] H. Konno and P.S. Lomdahl, The Birth-Death Stochastic Processes of Solitons in the 1D Benney Equation, J. Phys. Soc. Japan, 69, 1629-1641 (2000).

[13] B. P. Moghaddam and A. Aghili, A numerical method for solving Linear Non-homogenous Fractional Ordinary Differential Equation by using the operational matrices of the Bernstein polynomials, Applied mathematics and information sciences (AMIS), 6, 441-445 (2012). 
[14] N. Guzel, I. Emiroglu, C. Guler, F. Tasci and M. Sivri, A Solution Proposal to the Interval Fractional Transportation Problem, Applied mathematics and information sciences (AMIS), 6, 567-571 (2012).

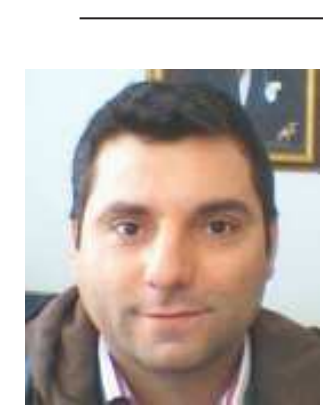

\section{Mehmet}

Ali Akinlar is an assistant professor of mathematics at Yildiz Technical University. He completed his M.Sc. at McMaster university and $\mathrm{Ph} . \mathrm{D}$. at UT, Arlington in 2005 and 2009, respectively. Upon completion of his Ph.D. he worked as a post-doctoral research associate at department of scientific computing at Florida State University under the supervision of Max Gunzburger. His research interests lie at applied and computational mathematics as well as solutions of all kinds of differential equations.

\section{Aydin}

Secer

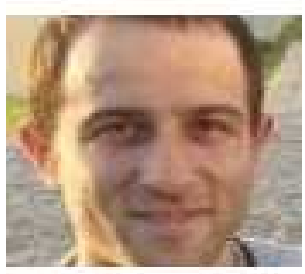

is an assistant professor of mathematics at department of mathematical engineering at Yildiz Technical University, Istanbul, Turkey. $\mathrm{He}$ is in the organizing committee of the International Conference: ICAAA2012. He is interested in scientific computing, computer programming,

numerical methods as well as differential and integral equations.

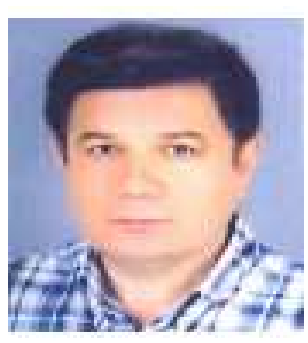

Mustafa Bayram is a full professor of mathematics at Yildiz Technical University. Currently he acts as the dean of the faculty of chemical and metallurgical engineering. His research interests include applied mathematics, solutions of differential equations, enzyme kinetics and mathematical biology. $\mathrm{He}$ raised many masters and Ph.D. students and is the author of many efficient research articles at prestigious research journals. He is the chief and founder editor of new trends in mathematical sciences journal. He also serves as an editor and reviewer for many outstanding mathematics journals. 